\title{
Scaling factors for production rates of in situ produced cosmogenic nuclides: a critical reevaluation
}

\author{
Tibor J. Dunai* \\ Isotopengeologie, Faculteit der Aardwetenschappen, De Boelelaan 1085, 1081 HV Amsterdam, The Netherlands
}

Received 20 August 1999; received in revised form 1 December 1999; accepted 1 December 1999

\begin{abstract}
New scaling factors are presented describing the altitude and latitudinal dependence of production rates for in situ produced cosmogenic nuclides. The new factors incorporate the influence of the non-dipole contributions to the geomagnetic field on the cosmic ray flux. The currently used scaling factors of Lal [Earth Planet. Sci. Lett. 104 (1991) 424 439] are based on the assumption of a dipole field. The overall strategy used in deriving the new scaling factors is, however, very similar to that of Lal and relies on the same and/or similar nuclear data. In this reevaluation, data are only used if the effective geomagnetic parameters (inclination, horizontal field strength) can be reconstructed for the time of measurement. The absorption free pathlengths $\Lambda$ for cosmic rays selected for this study are based on observational data at altitudes relevant for exposure age dating (sea level to $7000 \mathrm{~m}$ ). At sea level and latitudes between $20^{\circ}$ and $40^{\circ}$, the new factors are up to $18 \%$ lower and at high altitudes more than $30 \%$ higher than those of Lal. In addition to accounting for the influence of the effective geomagnetic field and providing more applicable estimates of $\Lambda$, the new factors also allow for correction for the significant deviations from the standard pressure-altitude relationship that exist in the atmosphere $(\leq 10 \%)$. (C) 2000 Elsevier Science B.V. All rights reserved.
\end{abstract}

Keywords: scale factor; cosmogenic elements; magnetic field; cosmochemistry

\section{Introduction}

The accuracy of exposure ages derived from the abundance of in situ produced cosmogenic nuclides depends on scaling factors for the latitude and altitude dependence of the cosmic ray flux. Currently, the scaling factors of Lal [1] are most widely used. These factors were derived from neu-

*Fax: +31-20-646-2457; E-mail: dunt@geo.vu.nl tron flux measurements and stars recorded on photographic emulsions in the years 1949-1955 [2-6]. The original aim in deriving scaling factors was to quantify cosmogenic nuclide production in the atmosphere [7-9]. Only later were these factors applied to in situ produced cosmogenic nuclides in surface rocks [1].

By transferring the application of the factors from the atmosphere to the surface of the Earth, however, approximations that are inherent in the scaling factors of Lal [1] become invalid. One approximation of Lal [1,7-9] is that the lateral variation of cosmic ray flux at sea level can be described by an axial geomagnetic dipole field. This 


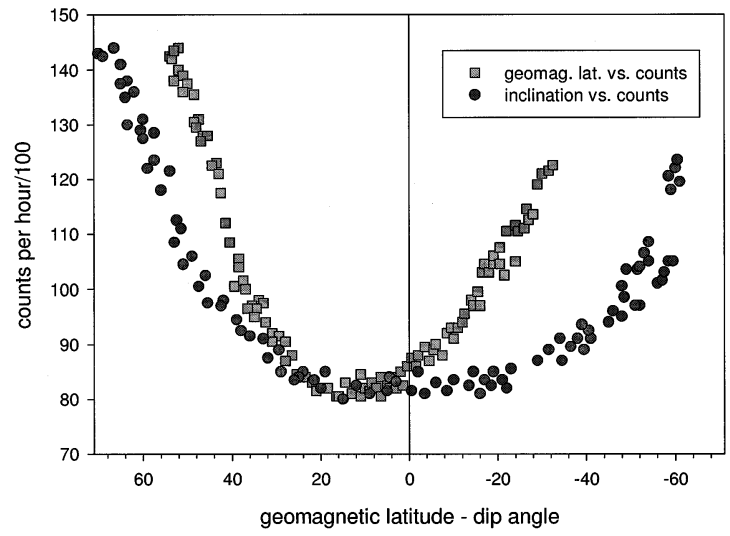

Fig. 1. Shipboard neutron flux data of Rothwell and Quenby [11] plotted vs. the geomagnetic latitude and the inclination dip angle, respectively. Squares are data plotted vs. latitude, the circles data plotted vs. inclination. Note that the geomagnetic latitude cannot be used as a parameter to describe the data, as the data are not symmetric with regard to the latitude equator. The data are, however, symmetric with regard to the dip equator, thus may be explained by the effective geomagnetic field. Further discussion is given in the text.

view was common within the physics community occupied with cosmic rays until about 1958 ([10], pp. 81-85). After 1958, triggered by the work of Rothwell and Quenby [11] (see also Fig. 1), it became generally accepted that only by considering the non-dipole field could the cosmic ray flux at sea level be described accurately [10]. Another approximation of Lal is that the values of absorption mean free path lengths for nuclear disintegrations derived at relatively high altitudes, 3.5-30 $\mathrm{km}$ [3-5], can be extrapolated to sea level. Such an extrapolation is, however, not strictly valid ([4], p. 1408, 2nd paragraph) and will inevitably lead to a too high value for the absorption free pathlength ([3], Table III and Fig. 4).

The new scaling factors rely in part on the same data as used by Lal and co-workers [7-9] and in part on additional data from measurements between 1948 and 1957. These data are evaluated using a similar approach as described by Lal [7], however, with two important differences: (1) now the effects of the geomagnetic field as described by International Geomagnetic Reference Field for the period of data acquisition are considered and (2) only absorption free pathlength determinations are used that were obtained at elevations relevant for exposure age dating utilizing in situ produced cosmogenic nuclides.

\section{Effects of the geomagnetic field on the cosmic ray flux}

As a first order approximation, the Earth's magnetic field can be described as a dipole field. This field partially shields the surface from primary cosmic ray particles. The primary particles (mainly protons) are charged and are therefore deflected when moving in a magnetic field. Only particles that have a momentum to charge ratio above a certain threshold can reach the surface of the Earth (for discussion see [10]). The cutoff rigidity $P(P=$ pcle $[\mathrm{GV}]$, where $p$ is the momentum of the particle $[\mathrm{GeV} / \mathrm{c}], c$ is velocity of light and $e$ the particle charge) of the geomagnetic field can be described for protons arriving vertical to the Earth's surface as (eq. 5 in [10]):

$P=\frac{M \cdot c}{4 R^{2}} \cdot \cos ^{4} \lambda$

where $M$ is the dipole moment [Wb], $R$ the radius of the Earth and $\lambda$ the geomagnetic latitude. Note that Eq. 1 is essentially the same formulation as described as 'trajectory tracing' of cosmic ray particles by Shea et al. (eq. 2 in [12]). Shielding is maximal at the geomagnetic equator and minimal at high latitudes. Above $60^{\circ}$ geomagnetic latitude the energy of protons that are permitted drops below the minimum energy of protons actually present in the cosmic radiation. Therefore the cosmic ray flux does not increase further above the $60^{\circ}$ latitude 'knee' [13].

Eq. 1 is, however, only satisfactory for studying effects of cosmic rays on a global scale. This is because the real geomagnetic field has a significant non-dipole component. Estimates for the contribution of the non-dipole field range between 10 and $25 \%$, depending on the methods used to derive the value [14]. There are also strong regional differences in the relative importance of the non-dipole field (see e.g. Fig. 2.3 in [14]). Therefore to investigate local effects, i.e. measuring spot nuclear disintegration rates that are essential in deriving scaling factors, the effective geomagnetic 
field at exactly the same location must be considered. Rothwell and Quenby [11] demonstrated that considering the effects of the non-dipole field, such as variations in the inclination with respect to the geomagnetic latitude, can explain observational data that do not fit the dipole model (Fig. 1). Following the formulation of Rothwell [15] a modified version of Eq. 1, considering the parameters describing the non-dipole field, can be written as follows:

$P=\frac{R}{4} \times \frac{H \cdot c}{\left(1+0.25 \cdot \tan ^{2} I\right)^{3 / 2}}$

where $H$ is the horizontal field intensity [T] and $I$ the inclination at the point of data acquisition. The effective values of $H$ and $I$ fully describe the geomagnetic field as is relevant for the modulation of the cosmic ray flux. Rothwell [15] showed that all shipboard neutron flux data published by 1958 , including those used by Lal (i.e. [6]), can be explained qualitatively by the effective geomagnetic field. This is in marked contrastto the dipole model which yields discrepancies in the effective geomagnetic latitude of up to $12^{\circ}$ $[11,16]$. Eq. 2 predicts that the cosmic ray equator (i.e. the line connecting minimum cosmic ray intensities in longitudinal profiles) is essentially identical to the inclination equator $\left(I=0^{\circ}\right)$.

In order to derive his scaling factors, Lal used a network of neutron flux measurements at various atmospheric depths (1030 g/ $\mathrm{cm}^{2}$ [6], $681 \mathrm{~g} / \mathrm{cm}^{2}$ [3], and $312 \mathrm{~g} / \mathrm{cm}^{2}$ [2], see [7]). All of these data show the effects of the real geomagnetic field, i.e. variable neutron fluxes for the identical geomagnetic latitudes (so-called longitude effect [3]). These deviations were acknowledged by the authors who presented the original data (caption of Fig. 5 in [3]; [6], p. 976). Consequently the shapes of the isobaric neutron flux curves used by Lal have a significant uncertainty (up to $20 \%$ difference in neutron fluxes at a given geomagnetic latitude, see Fig. 2 in [6]). The uncertainty is maximal at geomagnetic latitudes between $20^{\circ}$ and $40^{\circ}$ where the longitude effect on cosmic ray flux measurements is most pronounced [3,6]. The estimated uncertainty of the scaling factors of Lal of $\pm 10 \%$ [1] is identical to the uncertainty of the sea level neutron flux data from which the factors are derived.

\section{Attenuation of cosmic rays in the atmosphere}

Primary particles with a sufficiently high momentum to penetrate the Earth's magnetic field are further attenuated through interaction with atoms in the Earth's atmosphere. Consequently the cosmic ray flux and the number of interactions caused by cosmic rays decrease with atmospheric depth. This decrease is approximately exponential (e.g. [1]):

$N=N_{0} \cdot e^{-z / \Lambda}$

$N$ and $N_{0}$ are the number of observed particles/ interactions with and without attenuation, respectively, $z$ is the atmospheric depth in $\mathrm{g} / \mathrm{cm}^{2}$ and $\Lambda$ is the absorption mean free path length. The decrease is not strictly exponential because $\Lambda$ decreases with altitude [3]. The latitudinal dependence of the cut-off rigidity of the geomagnetic field described in Section 2 causes different values for $\Lambda$ at different geomagnetic latitudes, as absorption of the primary particles and their secondaries is energy-dependent [13].

It is evident from Eq. 3 that choosing the correct values for $\Lambda$ is crucial as even small differences will have a pronounced effect on the production rates at high altitudes. For high latitudes $\left(\lambda=42^{\circ}-65^{\circ}\right)$ and altitudes between sea level and $7000 \mathrm{~m}$, values reported for $\Lambda$ range between 120 and $141 \mathrm{~g} / \mathrm{cm}^{2}$ (Table 1) [3,17-21]. At low latitudes $\left(\lambda=0-21^{\circ}\right)$ and altitudes between 1680 and $5960 \mathrm{~m}$ all studies agree within the reported uncertainties with a value of $149 \mathrm{~g} / \mathrm{cm}^{2}$ for $\Lambda$ (Table 1) $[3,13,22,23]$. The range in high latitude values for $\Lambda$ is partially a result of the different detector designs. All data, except those of [18,19,22,23], were collected with $\mathrm{BF}_{3}$ proportional counters embedded in paraffin using a lead core or cover; paraffin to shield the detectors from locally produced neutrons [13,24], lead as a target to produce secondary neutrons, from interactions with high-energy neutrons (200-1000 MeV [24]), that are recorded. A discussion of the influence of de- 


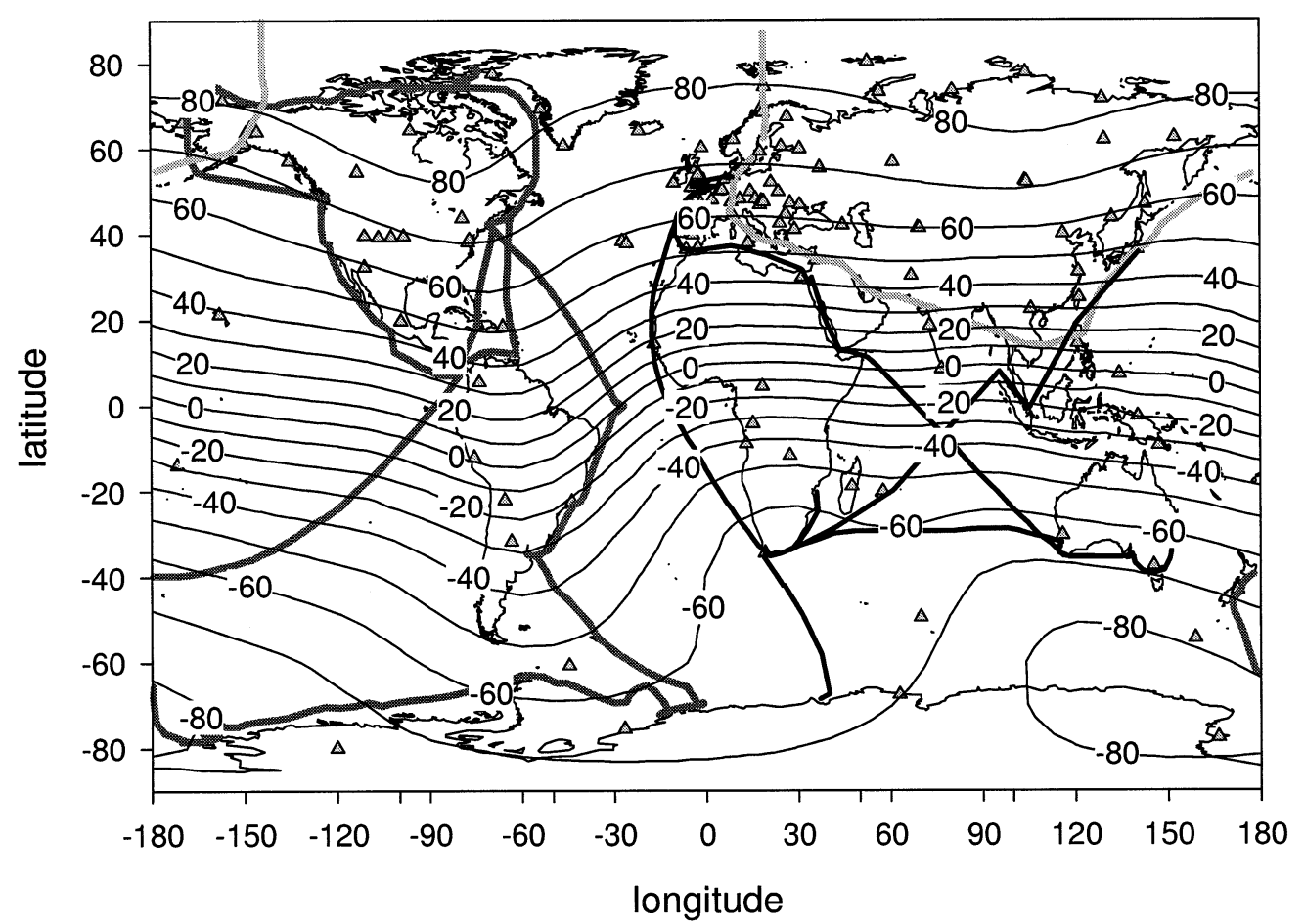

Fig. 2. Spatial distribution of published shipboard and airborne neutron flux data. To help to assess which data set is suitable for reevaluation, the surface inclination angle of the geomagnetic field in the spring of 1955 is plotted in isolines as a proxy for the non-dipole contributions to the geomagnetic field. The triangles give the positions of geomagnetic observatories that were active between 1955 and 1957 [42]. The light grey line represents airborne data locations of [17]. The location of shipboard data is given by the broad dark grey line (circum North America and Boston-Panama-New Zealand-Antarctic Sea-Buenos Aires-Boston [6], and the thin solid black lines (Europe-Cape Town-Mozambique [11,36], Japan-Cape Town-Antarctica [37], and EuropeMediterranean-Red Sea-Australia-Cape Town-Europe [38]). For discussion see text.

sign parameters on values for $\Lambda$ is given in [13,25]. Resolving this issue is beyond the scope of this paper. It is evident, however, that the chosen value of $\Lambda$ should be consistent with available experimental data. Thus, in deriving a neutron flux network to calculate scaling factors, the same counter design should be used to derive $\Lambda$ and the disintegration rates at and above sea level. The other, probably more important factor introducing scatter into the range for $\Lambda$ is the altitude range where the data were collected. The results of all studies at high latitudes [17-19,21], where data were acquired down to $1000 \mathrm{~m}$ above sea level or lower, agree within the reported uncertainties with a value for $\Lambda$ of $128 \mathrm{~g} / \mathrm{cm}^{2}$. Only the studies where the data were collected exclusively at higher altitudes $(>1900 \mathrm{~m})$ report higher values for $\Lambda\left(\sim 140 \mathrm{~g} / \mathrm{cm}^{2}\right)$. This observation is in agreement with the finding of Simpson and Uretz [3] that $\Lambda$ increases with increasing altitude.

The data of $[18,22,23]$ are internally consistent as all investigators used identical photographic emulsions (Ilford G5) and screening procedures. Furthermore, the photographic emulsions reliably record tracks of all nuclear disintegrations induced by cosmic rays, i.e. by protons, muons and secondary neutrons that have energies $>35$ $\mathrm{MeV}[22,26,27]$. Thus they give a good approximation of the entire range of cosmic ray interactions with solid matter.

Teucher [28] comments on the possibility that locally produced secondary neutrons (i.e. those produced/reflected at or below the air-surface interface [29]) are recorded as low energy events on the photographic emulsions and have an influence on the value of $\Lambda$ obtained. This view is in agree- 
ment with the calculations of Masarik and Reedy [29] that show that for $10-20 \mathrm{~g} / \mathrm{cm}^{2}$ on both sides of the air-surface interface the neutron flux is perturbed (see Fig. 1 in [29]). The neutron flux at both sides of the interface shows a relatively flat profile [29]. In all the photographic emulsion studies used here $[18,22,23]$ the emulsions were exposed within $1 \mathrm{~m}$ of the air-surface interface. Thus a portion of the neutron tracks recorded by the emulsions is a consequence of the perturbed neutron flux at the air-surface interface. Rocks that are sampled for exposure age dating record nuclear disintegrations (i.e. in situ produced cosmogenic nuclides) occurring at exactly the same interface. Hence, the values for $\Lambda$ derived from the photographic emulsion studies used in this paper $[18,22,23]$ probably best describe the altitude dependence of in situ production of cosmogenic nuclides at the air-surface interface.

Analogous to the photographic emulsion data the cloud chamber data were also obtained close to the air-surface interface [19] and record events of similar energy ( $>8 \mathrm{MeV}$ [19]) as photographic emulsions ( $>35 \mathrm{MeV}$ [27]). Hence the value of $132 \pm 4 \mathrm{~g} / \mathrm{cm}^{2}$ obtained by Brown [19] (Table 1) is equally valid to describe the altitude depen- dence of in situ production of cosmogenic nuclides at the air-surface interface as the values of $\Lambda$ obtained by [18]. Therefore, throughout this paper values of $130 \pm 4 \mathrm{~g} / \mathrm{cm}^{2}$ (mean of [18] and [19]) and $149 \pm 2 \mathrm{~g} / \mathrm{cm}^{2}$ [22,23] for high and low latitudes, respectively, will be used as guidelines for preferred values of $\Lambda$ for cosmic ray-induced nuclear disintegrations at the Earth's surface. Note that the value for $\Lambda$ obtained with the data collected with $\mathrm{BF}_{3}$ proportional counter at high latitudes and low altitudes (i.e. $130 \mathrm{~g} / \mathrm{cm}^{2}$ [17], Table 1) is in agreement with the photographic emulsion and cloud chamber data.

The above values for $\Lambda$ describe the interactions of the integrated cosmic ray flux, i.e. the sum of all interactions induced by protons, muons and secondary neutrons. Reaction cross-sections for fast protons and neutrons are similar $[1,30]$. These particles also dominate the cosmic ray flux and nuclear interactions producing cosmogenic nuclides. Thus the values for $\Lambda$ obtained above are mostly relevant for the proton and neutron components (nucleonic component) and their interactions. Some target elements in rocks $(\mathrm{O}, \mathrm{Na}, \mathrm{Mg}$, $\mathrm{Al}, \mathrm{Si}$ ), however, have a large cross-section for negative muon capture producing cosmogenic nu-

Table 1

Absorption free pathlength $\Lambda$ in the atmosphere determined at altitudes $<9000 \mathrm{~m}$ down to sea level

\begin{tabular}{|c|c|c|c|c|}
\hline Geomagnetic latitude $\lambda$ & Source & Method & Altitude range & $\begin{array}{l}\Lambda \\
\left(\mathrm{g} / \mathrm{cm}^{2}\right)\end{array}$ \\
\hline $60-65^{\circ}$ & [17] & $\mathrm{BF}_{3}$ counter $^{\mathrm{a}}$ & $\begin{array}{l}1000-7000 \mathrm{~m} \\
4300-9000 \mathrm{~m}\end{array}$ & $\begin{array}{l}130 \\
138\end{array}$ \\
\hline $52^{\circ}$ & {$[3]$} & $\mathrm{BF}_{3}$ counter $^{\mathrm{a}}$ & $2500-5000 \mathrm{~m}$ & $141 \pm 2$ \\
\hline $47-50^{\circ}$ & [18] & emulsion $^{\mathrm{b}}$ & $150-3774 \mathrm{~m}$ & $127 \pm 3$ \\
\hline $47-50^{\circ}$ & [19] & cloud chamber ${ }^{\mathrm{c}}$ & $0-3230 \mathrm{~m}$ & $132 \pm 4$ \\
\hline $44^{\circ}$ & [20] & $\mathrm{BF}_{3}$ counter $^{\mathrm{a}}$ & $1900 \mathrm{~m}$ & $\begin{array}{l}125 \pm 10^{\mathrm{d}} \\
140 \pm 2^{\mathrm{e}}\end{array}$ \\
\hline $42-45^{\circ}$ & {$[21]$} & $\mathrm{BF}_{3}$ counter ${ }^{\mathrm{a}}$ & $260-4300 \mathrm{~m}$ & $132 \pm 15$ \\
\hline $42^{\circ}$ & & & $260 \mathrm{~m}$ & $121 \pm 7$ \\
\hline $21^{\circ}$ & {$[22]$} & emulsion $^{\mathrm{b}}$ & $2630-5350 \mathrm{~m}$ & $149 \pm 2$ \\
\hline $0^{\circ}$ & [13] & $\mathrm{BF}_{3}$ counter $^{\mathrm{a}}$ & $3400-4400 \mathrm{~m}$ & $149 \pm 9$ \\
\hline $0^{\circ}$ & {$[3]$} & $\mathrm{BF}_{3}$ counter $^{\mathrm{a}}$ & $3000-4200 \mathrm{~m}$ & $145 \pm 9$ \\
\hline $0^{\circ}$ & [23] & emulsion $^{\mathrm{b}}$ & $1680-5960 \mathrm{~m}$ & $149 \pm 2^{\mathrm{f}}$ \\
\hline
\end{tabular}

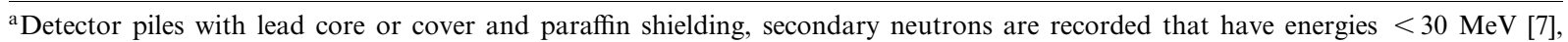
the primary particles producing the secondaries have energies $\sim 200 \mathrm{MeV}-1 \mathrm{GeV}$ ([24] and references therein).

${ }^{\mathrm{b}}$ Ilford G5 emulsions, $\Lambda$ as determined from stars with $\geq 3$ prongs, i.e. tracks from particle with energies $\geq 35 \mathrm{MeV}$ [27].

${ }^{\mathrm{c}}$ Cloud chamber filled with 5 bar argon, spallation events induced by particles that have energies $>8 \mathrm{MeV}$ are recorded [19].

${ }^{\mathrm{d}}$ Low energy neutrons recorded with cadmium and paraffin shielding, $<0.5 \mathrm{eV}[20]$.

e'Mormal' $\mathrm{BF}_{3}$ counter used.

${ }^{\mathrm{f}}$ Maximum error, own estimate by comparing counting statistics and curve fit to results of [22]. 
clides that are frequently used for exposure age dating (e.g. ${ }^{10} \mathrm{Be},{ }^{26} \mathrm{Al},{ }^{21} \mathrm{Ne},{ }^{22} \mathrm{Ne}$ [31]). Thus production of those nuclides can be appreciably influenced by the muon component. In the case of ${ }^{10} \mathrm{Be}$ and ${ }^{26} \mathrm{Al}$ the reported contribution by muon capture varies between $1-3 \%$ and $17 \pm 9 \%$ (in quartz [32-34]). The absorption free pathlength for muons is larger than that of the nucleonic component. The only reliable value that is currently available for $\Lambda$ for muons in the atmosphere is $247 \mathrm{~g} / \mathrm{cm}^{2}$ and is valid between sea level and $12 \mathrm{~km}[31,35]$. The latitude dependence below $\sim 5 \mathrm{~km}$ seems to closely parallel that of the nucleonic component ([32] and references therein).

Calculating the values of $\Lambda$ from the scaling factors of Lal (Table 2 in [1]) for altitudes below $3500 \mathrm{~m}$, one obtains 135 and $157 \mathrm{~g} / \mathrm{cm}^{2}$ for high and low latitudes, respectively. For altitudes $>3500 \mathrm{~m}$ the corresponding values are 157 and $174 \mathrm{~g} / \mathrm{cm}^{2}$. While the relative latitude effect is about the same order as implied by the photographic emulsion studies [18,22,23], the absolute values are on the high side. All available experimentally derived values of $\Lambda$ for low altitudes at low latitudes are lower $[13,22,23]$ than those used by Lal. The same is true for the experimentally derived values of $\Lambda$ for low altitudes at high latitudes [17-19,21]. Also for altitudes $>3500$ none of the studies listed in Table 1 supports the even higher values of $\Lambda$ inherent in Lal's scaling factors, although four studies extended to altitudes of $5000 \mathrm{~m}$ and higher $[3,17,22,23]$.

\section{Deriving the new scaling factors}

\subsection{The data for the new neutron flux network}

The overall approach used to derive new scaling factors for cosmic ray-induced nuclear disintegrations in this study is very similar to that of Lal $[1,7]$. The main difference in this study lies in the selection of isobaric neutron flux data and the choice of $\Lambda$. For the new scaling factors I use only data which can be reevaluated by reconstructing the effective geomagnetic field at the time of the neutron flux measurement, i.e. data for which the exact location for each measure- ment was published. Furthermore the selected data sets extend beyond the high latitude 'knee' of the isobaric geomagnetic latitude curves to allow normalization of the data. The normalization is important, because although principally the same kind of neutron flux detector was used in all studies $\left(\mathrm{BF}_{3}\right.$ proportional counters embedded in paraffin and lead shielding) the counters are not identical and deviate in design. Thus, although absolute counting rates cannot be compared directly, normalized data can be compared (see [13], pp. 941-944). Fig. 2 shows the spatial distribution of available neutron flux data at sea level and altitudes $<5000 \mathrm{~m}$. Of the available shipboard data, those of Rose et al. [6] are by far the most extensive. Coverage includes both the northern and southern hemispheres beyond the latitude knee $\left(>60^{\circ}\right.$ latitude i.e. $>80^{\circ}$ inclination for a dipole field, see Section 4.2). The data set is therefore ideal for reevaluation and should deliver a reliable sea level neutron flux curve. The other sea level data sets [11,36-38] do not extend beyond the latitude knee, thus can only be used for qualitative arguments as done by Rothwell [15]. The data of Kodoma [37] mimic a knee but are the result of high latitude data collection from an area with abnormally shallow, and spatially rather uniform inclination (Fig. 2).

Sandström [17] reports the only airborne data set that is suitable for reevaluation (Fig. 2). Data were collected at $600 \mathrm{~g} / \mathrm{cm}^{2}$ atmospheric depth ( $\sim 4300 \mathrm{~m}$ ) and cover a wide latitudinal and longitudinal range. Other data sets either do not cover a wide enough latitudinal range [3] and/or can not be reevaluated due to missing coordinates $[2,3]$.

Following the reasoning given in Section 3, I chose the mean absorption free path length of Sandström of $130 \mathrm{~g} / \mathrm{cm}^{2}$ [17] to link the sea level neutron flux data of Rose et al. [6] with the high altitude data of Sandström [17]. $\Lambda$ has been determined from measurements derived at altitudes from $1000 \mathrm{~m}$ to $7000 \mathrm{~m}$ [17], thus providing an average $\Lambda$ for that altitude range. Sandström [17] also reports a higher value for $\Lambda$ derived using data between 4300 and $9000 \mathrm{~m}$. The higher value, $138 \mathrm{~g} / \mathrm{cm}^{2}$, is in agreement with the high altitude 
data of [3] (Table 1). The lower value of $130 \mathrm{~g} /$ $\mathrm{cm}^{2}$ is used here because it describes the altitude dependence in the depth range relevant for most studies utilizing in situ produced cosmogenic nuclides. Moreover, this value for $\Lambda$ agrees well with

A

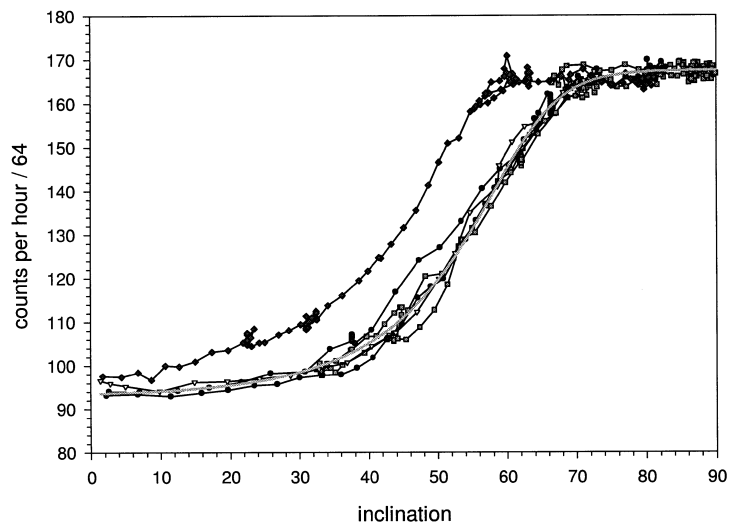

B

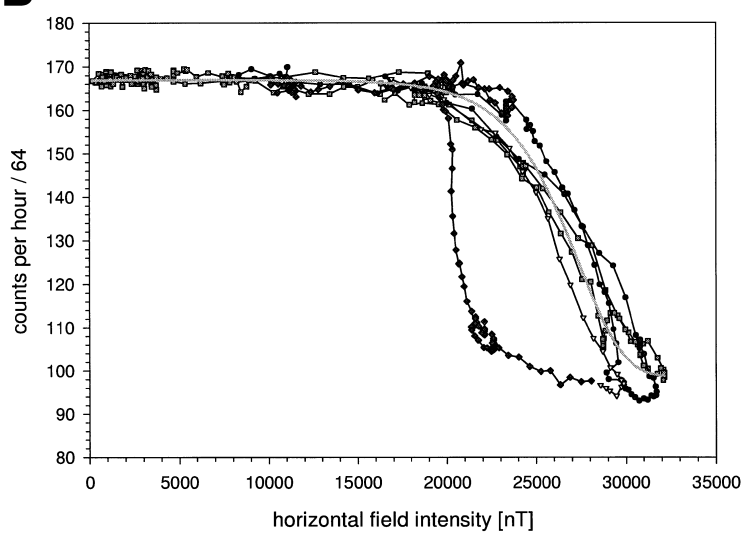

C

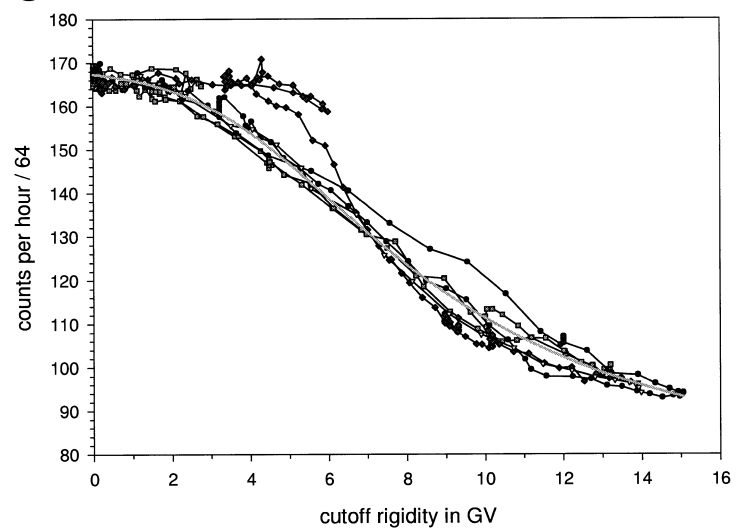

photographic emulsion [18] and cloud chamber data [19] that were obtained at altitudes between sea level and $3500 \mathrm{~m}$ (mean of $130 \pm 4 \mathrm{~g} / \mathrm{cm}^{2}$ ). Thus the value of $130 \mathrm{~g} / \mathrm{cm}^{2}$ appears a good linear approximation for values of $\Lambda$ for nuclear disintegrations induced by cosmic rays between sea level and $7000 \mathrm{~m}$ at high latitudes. Based on the variation seen in high latitude photographic emulsion and cloud chamber data (see Section 3 ) the estimated uncertainty in $\Lambda$ used in this paper is $\pm 3 \%$.

The shipboard data of Rose et al. [6] were collected during a period of low solar activity (1954 55) and the airborne data of Sandström [17] were collected at a time of increased solar activity (February 1957) [10]. While this affects the absolute counting rates, the overall shape of the neutron flux curves at large atmospheric depth $(>600 \mathrm{~g} /$ $\mathrm{cm}^{2}$ ) is not affected. This conclusion is based on the observation that mountain altitude $(\sim 2000$ m) neutron detectors show the same relative intensity change as sea level neutron detectors during a solar cycle [39]. Thus, using normalized neutron flux data will eliminate not only detector biases but also the neutron flux modulation during a solar cycle.

The geomagnetic field parameters at the time and location of the neutron flux measurements are reconstructed using the GEOMAGIX software of John Quinn of the USGS National Geomagnetic Information center (downloadable at http://geomag.usgs.gov). Models Dgrf-50 and Dgrf-55 were used that are based on observation station data only [40]. Discussion of the model accuracies and information about the distribution

Fig. 3. Shipboard neutron flux data of Rose et al. [6] plotted vs. (A) inclination $I,(\mathrm{~B})$ horizontal field intensity $H$ and (C) the cut-off rigidity of the geomagnetic field $P$. Circum North-America data (squares), Boston-Panama-New Zealand-Antarctic $\left(180-175^{\circ} \mathrm{W}\right)$ data (circles), Antarctic (175- $0^{\circ}$ W)-South Atlantic data (diamonds) and North Atlantic data (triangles down). The results of non-linear regression (five parameter sigmoidal) of all the data, excluding the Antarctic $\left(175-0^{\circ} \mathrm{W}\right)-$ South Atlantic data, are given as grey curves. The data format for the counting rate (counts per hour/64) is as reported in the original study by [6] and should be read as arbitrary units. For further discussion see text. 
of observational data in the 1950 s can be found in $[41,42]$.

\subsection{Constructing the neutron flux network}

In order to account for the non-dipole components of the Earth's magnetic field the neutron flux is fitted to parameters describing the field. Parameters considered here are the inclination $I$, horizontal field intensity $H$ and the cut-off rigidity $P$. It is clear from Fig. 3 that the majority of data can well be fitted to all three parameters. Only the data from the Antarctic Sea and the South Atlantic are displaced with respect to the other data in all three cases. This is because the highly anomalous geomagnetic field (see e.g. Fig. 2) in this area cannot be reconstructed satisfactorily from the few observational data of the geomagnetic field available for this area in the 1950s ([41,42], John Quinn, personal communication). The results from this area are therefore excluded from further consideration. Non-linear regression (five parameter sigmoidal, SigmaPlot $4.0^{\circledR}$ software) of the remaining data, treated as one population, yields coefficients of correlation $\left(r^{2}\right)$ of 0.994 , 0.993 and 0.972 for values of $I, P$ and $H$ as variables, respectively. The corresponding standard errors of estimates $(\sigma)$ are $\sim 2 \%$ for $I$ and $P$ and $\sim 4.5 \%$ for $H$. The standard errors of estimates of $I$ and $P$ compare well with the reported reproducibility of neutron flux measurements $( \pm 1.8 \%$ [43]) and the globally averaged uncertainty of reconstructed geomagnetic field parameters $\left(\sigma=\sim 1 \%\right.$ or $1^{\circ}$, whatever applicable [44]). Therefore the sigmoidal functions shown in Fig. 3 describe the sea level neutron flux data very well. That the close fits of observational data to $I$ and $P$ are so similar can easily be explained by Eq. 2 where changes of $P$ are most sensitive to changes of $I$. As the goal of this paper is to derive scaling factors that can be easily related to geographic coordinates, I chose the inclination $I$ as variable describing the neutron flux at sea level. Consequently the airborne neutron flux data [17] are also fitted to the inclination $I$ in an identical manner (Fig. 4) $\left(r^{2}=0.993, \sigma \sim 2 \%\right)$.

The remaining step in constructing the neutron flux network is to normalize the neutron curves

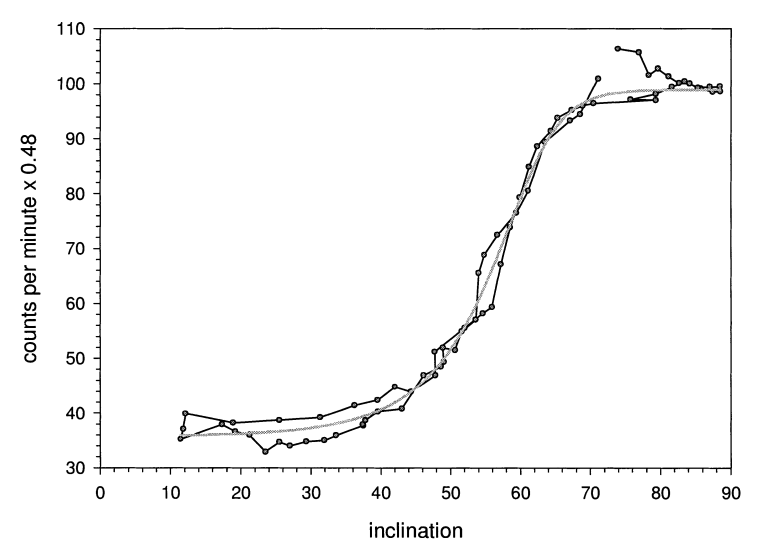

Fig. 4. Airborne neutron flux data of Sandström [17] plotted vs. the inclination $I$. The result of non-linear regression (five parameter sigmoidal) is displayed as a grey curve. Six anomalously high data points [17] are not included in the regression. The data format for the counting rate (counts per minute $\times 0.48$ ) is as reported in the original study by [17] and should be read as arbitrary units.

(Figs. 3 and 4). The sea level $\left(1030 \mathrm{~g} / \mathrm{cm}^{2}\right.$ or 1013 mbar) neutron flux curve is normalized to $I=90^{\circ}$. The resulting function $N_{1030}(I)$ (with $N_{1030}(90)=1$ ) can be described as a five parameter sigmoidal function $\left(r^{2}=0.994, \sigma \approx 2 \%\right.$, parameters $A, B, C$, $X$ and $Y$ in Table 2):

$$
N_{1030}(I)=Y+\frac{A}{\left[1+e^{-\left(\frac{1-X}{B}\right)}\right]^{C}}
$$

The high altitude neutron flux curve $\left(600 \mathrm{~g} / \mathrm{cm}^{2}\right.$ or 590 mbar, $4300 \mathrm{~m}$; Fig. 4) is connected to the sea level curve at $I=75^{\circ}$ using $\Lambda=130 \mathrm{~g} / \mathrm{cm}^{2}$ ( $\Lambda$ was obtained at $I \sim 75^{\circ}$ [17]), resulting in $N_{600}(I)$. From that network, the inclination dependence of $\Lambda$ can be derived by writing Eq. 3 as:

$N_{600}(I)=N_{1030}(I) \cdot e^{(1030-600) / \Lambda(I)}$

thus:

$$
\Lambda(I)=\frac{430}{\ln \left[\frac{N_{600}(I)}{N_{1030}(I)}\right]}
$$

The resulting $\Lambda(I)$ is shown in Fig. 5. Note that 
the values for $\Lambda$ at low inclination (i.e. low latitude) are in perfect agreement with observational low latitude data (Table 1, [13,22,23], although the $N_{600}(I)$ was linked at high latitudes. Thus $N_{600}(I)$ is consistent with the observational low altitude data for $\Lambda$ collected at high and low latitudes (Table 1). $\Lambda(I)$ can be described by a five parameter sigmoidal function $\left(r^{2}=0.9995\right.$, $\sigma \approx 0.2 \%$, parameters $a, b, c, x$ and $y$ in Table 2$)$ : $\Lambda(I)=y+\frac{a}{\left[1+e^{-\left(\frac{1-x}{b}\right)}\right]^{c}}$

Eq. 3 can be written as:

$N(z, I)=N_{1030}(I) \cdot e^{z(h) / \Lambda(I)}$

where $N_{1030}(I)$ and $\Lambda(I)$ are the five parameter sigmoidal functions derived earlier (Eqs. 4 and 7 ) and $z(h)$ is the value of the difference in atmospheric depth at height $h$ compared to sea level. $z(h)$ can be derived from functions describing the pressure gradient in the atmosphere (for standard atmosphere, see Appendix). It is advisable to use realistic estimates of surface temperatures to calculate $z(h)$ as there are significant differences in calculated elevations for the same pressure for different average surface temperatures. For example the isobars in the tropics $\left(25^{\circ} \mathrm{C}\right)$ are in the order of $4-5 \%$ higher than those in temper-

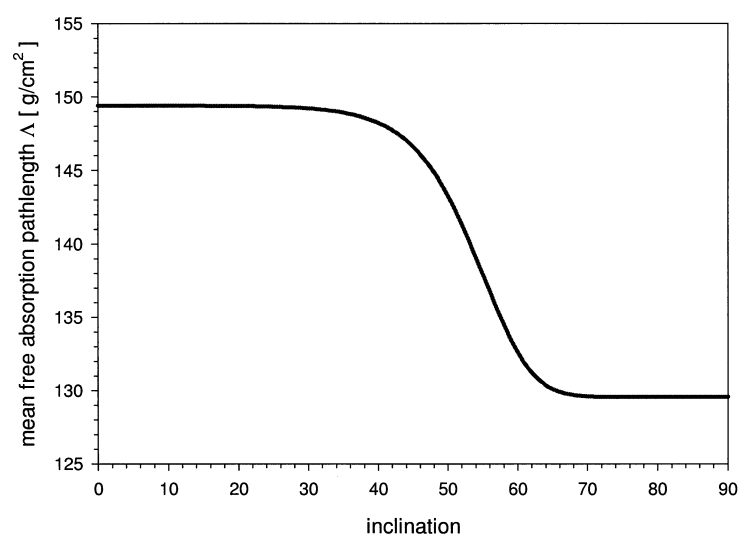

Fig. 5. The mean free absorption path length as a function of inclination $I$. For further discussion see text.
Table 2

Nuclear disintegration rates induced by cosmic rays normalized to sea level and $90^{\circ}$ inclination. Parameters for Eqs. 4 and 7

\begin{tabular}{lllll}
\hline \multicolumn{2}{ll}{ Coefficients for } & $N_{1030}(I)$ & & \multicolumn{2}{l}{ Coefficients for $\Lambda(I)$} \\
\cline { 1 - 2 }$B$ & 0.4450 & & $a$ & 19.85 \\
$B$ & 4.1703 & & $b$ & -5.430 \\
$C$ & 0.3350 & & $c$ & 3.590 \\
$X$ & 62.698 & & $x$ & 62.050 \\
$Y$ & 0.5555 & $y$ & 129.55 \\
\hline
\end{tabular}

ate regions $\left(15^{\circ} \mathrm{C}\right)[45,46]$. Furthermore it should be noted that the average sea level pressure in some areas (mostly oceanic) can deviate by up to 10 mbar from standard sea level pressure $[47,48]$ thus changing production rates up to $\sim 7 \%$.

As values for $N$ are normalized, $N(z, I)$ is the scaling function for nuclear disintegration rates in the atmosphere and can be used to relate production rates of in situ produced cosmogenic nuclides. The parameters for the sigmoidal functions are given in Table 2. Eq. 8 is strictly only valid for the cosmogenic production by the nucleonic component of the cosmic rays (protons and secondary neutrons, see Section 3). For cosmogenic nuclides with an appreciable production due to muon capture reactions Eq. 8 can be modified to include that production:

$N(z, I)=$

$N_{1030}(I) \cdot e^{z(h) / \Lambda(I)} \cdot(1-x)+N_{1030}(I) \cdot e^{z(h) / \Lambda_{\mu}} \cdot x$

where $x$ is the fraction of the cosmogenic nuclide produced by muon capture at sea level and $\Lambda_{\mu}$ the absorption free pathlength of muons in the atmosphere. A detailed description of how to derive scaling factors for use in exposure age dating is given in the Appendix.

The long term ( $>10000-20000$ years) timeaveraged geomagnetic field can be described by a geocentric axial dipole field (see e.g. [14]). Therefore the inclination $I$ can be translated into geomagnetic latitude (eq. 3.3.4. in [14]):

$\tan I=2 \tan \lambda$ 


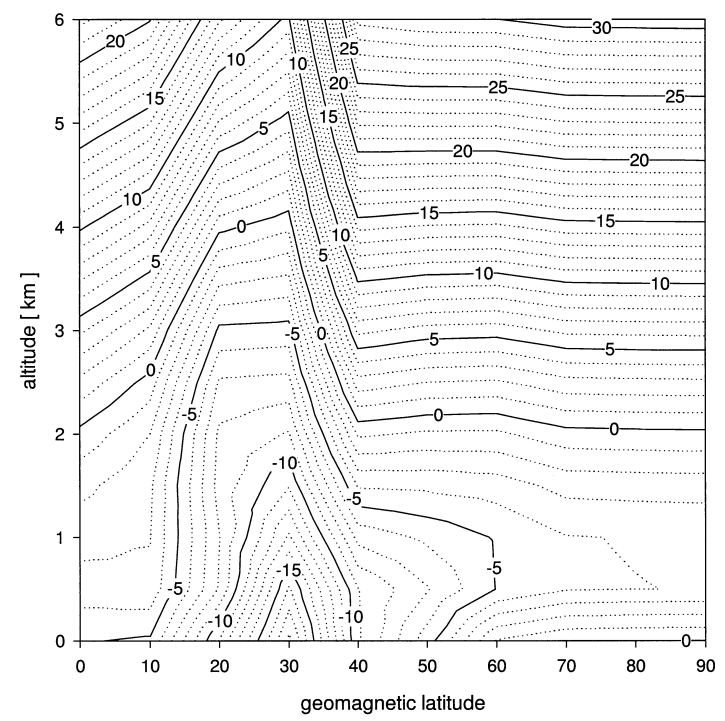

Fig. 6. Percent difference of the new scaling factors compared to those of Lal ([1], Table 2). The differences were calculated assuming a standard atmosphere pressure-altitude relationship, i.e. $15^{\circ} \mathrm{C}$ surface temperature. Use of more realistic estimates would reduce differences at high altitudes in the tropics $(-\sim 5 \%)$ and increase differences at high altitudes in the boreal areas $(+\geq 5 \%)$.

Thus when comparing measurements of in situ produced cosmogenic nuclides in surfaces older than 20000 years the geographic latitude may be used to calculate the inclination $I$ used in Eqs. 8 and 9. For samples younger than 20000 years the time-averaged geomagnetic field is not a geocentric axial dipole field [14]. Therefore the real value of $I$ must be approximated using proximal paleomagnetic records.

\section{Discussion}

The relative differences between the new scaling factors and the commonly used factors of Lal [1] are presented in Fig. 6. The largest differences are found between $20^{\circ}$ and $40^{\circ}$ latitude close to sea level and at high altitudes. In these regions the differences exceed the $10 \%$ error estimate given by [1]. As discussed earlier the deviation between $20^{\circ}$ and $40^{\circ}$ latitude is caused by unresolved nondipole field effects in the data used by Lal (e.g.
$[3,6])$. The deviations at high altitude are due to the higher values of $\Lambda$ used by Lal which are at odds with observational data from the altitude range relevant for exposure age dating (sea level to $7000 \mathrm{~m}$, see Section 3).

The new scaling factors leave some space for future improvements. The sea level neutron flux curve is rather well constrained $(\sim 2 \%, 1 \sigma$, see Section 4.2). Thus errors introduced by the scaling factors on calculating production rates at sea level will be of the same order $(\sim 2 \%)$. However, the estimated $3 \%$ uncertainty of $\Lambda$ is magnified for high altitudes as $\Lambda$ is used in the exponent of Eq. 6. Therefore the overall uncertainty of calculated production rates using the new scaling factors increases with increasing altitude (e.g. by $\sim 6 \%$ at $3000 \mathrm{~m}$, by $\sim 10 \%$ at $5000 \mathrm{~m}$ and by $\sim 20 \%$ at $7000 \mathrm{~m}$ ). The $10 \%$ error estimate given by Lal for his scaling factors [1] includes only the uncertainty of his sea level neutron flux curve (see end of Section 2). If an uncertainty is assumed for $\Lambda$, the error estimate for the currently used scaling factors of [1] must also increase with increasing altitude (similar to above values, if $\Lambda$ has an uncertainty of e.g. $3 \%$ ). It is evident from the discussion above that future experimental work should be directed at accurately constraining $\Lambda$ as a function of altitude and latitude.

\section{Conclusions}

The non-dipole components of the Earth's magnetic field contribute up to $20 \%$ to the total field; thus they must be considered when studying short-term effects of cosmic rays. Only over time scales exceeding 20000 years can the geocentric axial dipole hypothesis be applied. Thus, neutron flux measurements that are used to derive scaling factors for cosmogenic nuclide production have to be evaluated using the effective geomagnetic field parameters such as inclination and horizontal field strength. Following this approach neutron, flux data are reevaluated to derive scaling factors.

There are significant differences between the new scaling factors and those of Lal [1]. Between sea level and $3500 \mathrm{~m}$ altitude, the first order dif- 
ferences $(\leq 18 \%)$ are the result of variations between idealized dipole assumption and the effective geomagnetic field. At higher altitudes, the larger values for $\Lambda$ used by Lal result in calculated production rates that are lower than the new scaling factors by up to $\sim 30 \%$.

For samples younger than 20000 years the average paleo-inclination in the region should be determined if possible, since deviations from the long-term averaged dipole field, which can persist over this time range, will induce significant errors.

The formula for the new scaling factors allows correction for actual deviations from the standard atmosphere assumption. The errors that may be avoided by accounting for the actual pressure-altitude relationship are in the order of $5-10 \%$. The proposed new scaling factor functions will allow a better worldwide comparability of exposure ages and production rate calibrations than can be achieved using the scaling factors of Lal [1]. At sea level the uncertainty of the new factors is about $2 \%$, at higher altitudes the uncertainty of $\Lambda$ takes effect and the associated uncertainty increases significantly. Further work is required to better constrain $\Lambda$.

\section{Acknowledgements}

This study profited from critical reviews of Paul Bierman, Devendra Lal, Joe Licciardi, Jozef Masarik, Samuel Niedermann, Rainer Wieler and an anonymous reviewer, who are gratefully acknowledged. I am grateful for discussions and moral support during the struggle by friends and colleagues at the VU. This is NSG publication number 991103.[R $\boldsymbol{V}$ ]

\section{Appendix}

The following procedure provides a step by step method to calculate production rates using the new scaling factors.

(A) Calculate the inclination $I$.

For surfaces older than 20000 years the inclination $I$ can be calculated from the geographic latitude $\lambda$ using the relationship (eq. 3.3.4. in [14]):

$$
\tan I=2 \tan \lambda
$$

(eq. 10 in text)

(B) Calculate the long-term average atmospheric pressure $p$ [mbar] at the sampling point. Here I give the equation for the standard atmosphere as adopted by the International Civil Aviation Organization (ICAO) as an approximation for the real atmosphere (eq. 54 in [46]). Other equations can be used if preferred:

$p=p_{0}\left(1-\frac{\beta_{0} h}{T_{0}}\right)^{\frac{g_{0}}{R_{\mathrm{d}} \beta_{0}}}$

$p_{0}$ is the pressure at sea level (standard pressure 1013.25 mbar, actual value might deviate in some regions $[45,48]), \beta_{0}$ the temperature decrease with elevation $(6.5 \mathrm{mK} / \mathrm{m}), h$ is the altitude in meters, $T_{0}$ the temperature in Kelvin at sea level (standard temperature $15^{\circ} \mathrm{C}=288.15 \mathrm{~K}$, use an appropriate value depending on the climatic region), $g_{0}$ the standard sea level value of the acceleration due to gravity $\left(9.80665 \mathrm{~m} / \mathrm{s}^{2}\right), R_{\mathrm{d}}$ the gas constant $(287.05 \mathrm{~J} / \mathrm{kg} / \mathrm{K})$.

(C) Calculate the atmospheric depth $d\left[\mathrm{~g} / \mathrm{cm}^{2}\right]$ at sampling site.

$$
d=10 p / g_{0}
$$

Calculate the atmospheric depth $d_{0}$ at sea level:

$d_{0}=10 p_{0} / g_{0}$

(NB, using the above values of $p_{0}$ and $g_{0}$ yields $d_{0}=1033 \mathrm{~g} / \mathrm{cm}^{2}$, i.e. a value $0.3 \%$ higher than the sea level $d_{0}$ in the text $\left(1030 \mathrm{~g} / \mathrm{cm}^{2}\right)$. This arises from the use of the rounded value for $g_{0}$ of 9.81 $\mathrm{m} / \mathrm{s}^{2}$ in the original studies in the $1950 \mathrm{~s}$. It has no influence on the scaling factors because they are normalized and describe relative changes only.)

(D) Calculate the difference in atmospheric depth between sampling site and sea level $z(h)$ :

$$
z(h)=d_{0}-d
$$

(E) Calculate the sea level neutron flux normal- 


$$
\begin{aligned}
& \text { ized to } I=90^{\circ}, N_{1030}(I) \text { : } \\
& N_{1030}(I)=Y+\frac{A}{\left[1+e^{\left.-\left(\frac{1-X}{B}\right)\right]^{C}}\right.}
\end{aligned}
$$

(eq. 4 in text)

$(A=0.4450, \quad B=4.1703, \quad C=0.3350, \quad X=62.698$, $Y=0.5555$, Table 2).

(F) Calculate the mean absorption free pathlength $\Lambda(I)$ :

$$
\Lambda(I)=y+\frac{a}{\left[1+e^{-\left(\frac{I-x}{b}\right)}\right]^{c}} \quad(\text { eq. } 7 \text { in text })
$$

$(a=19.85, \quad b=-5.430, \quad c=3.590, \quad x=62.050$, $y=129.55$, Table 2).

(G) Calculate the scaling factor $N(z, I)$ :

$$
N(z, I)=N_{1030}(I) \cdot e^{z(h) / \Lambda(I)} \quad(\text { eq. } 8 \text { in text })
$$

or in the case of cosmogenic nuclides which have an appreciable contribution by muon capture reactions (for list of nuclides and target elements see Table 6 in [31]):

$$
\begin{aligned}
& N(z, I)= \\
& \quad N_{1030}(I) \cdot e^{z(h) / \Lambda(I)} \cdot(1-x)+N_{1030}(I) \cdot e^{z(h) / \Lambda_{\mu}} \cdot x
\end{aligned}
$$

(eq. 9 in text)

$x$ is the fraction of the cosmogenic nuclide produced by muon capture at sea level and $\Lambda_{\mu}$ the absorption free pathlength of muons in the atmosphere $\left(247 \mathrm{~g} / \mathrm{cm}^{2}\right)$.

(H) Calculate the production rate of a cosmogenic nuclide at the sampling site.

The production rates $P_{\text {nuclide }}$ of in situ produced cosmogenic nuclides are usually reported as values for sea level and high latitudes $\left(\lambda \geq 60^{\circ}\right.$, i.e. $I \geq 74^{\circ}$ ). Therefore the production rate at the sampling site is easily calculated by multiplying
$P_{\text {nuclide }}$ by $N(z, I)$ :

$P_{\text {nuclide }}(z, I)=P_{\text {nuclide }} \cdot N(z, I)$

\section{References}

[1] D. Lal, Cosmic ray labeling of erosion surfaces: in situ nuclide production rates and erosion models, Earth Planet. Sci. Lett. 104 (1991) 424-439.

[2] J.A. Simpson, Neutrons produced in the atmosphere by the cosmic radiations, Phys. Rev. 83 (1951) 1175-1188.

[3] J.A. Simpson, W.C. Fagot, Properties of the low energy nucleonic component at large atmospheric depth, Phys. Rev. 90 (1953) 1068-1072.

[4] R.K. Soberman, High-altitude cosmic ray neutron intensity variations, Phys. Rev. 102 (1956) 1399-1409.

[5] J.J. Lord, The altitude and latitude variation in the rate of occurrence of nuclear disintegrations in the Stratosphere by cosmic rays, Phys. Rev. 81 (1951) 901-909.

[6] D.C. Rose, K.B. Fenton, J. Katzman, J.A. Simpson, Latitude effect of the cosmic ray nucleon and meson components at sea level from the Arctic to the Antarctic, Can. J. Phys. 34 (1956) 968-984.

[7] D. Lal, P.K. Malhotra, B. Peters, On the production of radioisotopes in the atmosphere by cosmic radiation and their application to meteorology, J. Atmos. Terrest. Phys. 12 (1958) 306-328.

[8] D. Lal, B. Peters, Cosmic ray produced isotopes and their application to problems in geophysics, in: J.G. Wilson, S.A. Wouthuysen (Eds.), Progress in Elementary Particle and Cosmic Ray Physics, Vol. 6, North Holland, Amsterdam, 1962, pp. 77-243.

[9] D. Lal, B. Peters, Cosmic ray produced radioactivity on earth, in: S. Flugg (Ed.), Handbook of Physics, Vol. 46/2, Springer, Berlin, 1967, pp. 551-612.

[10] W.R. Webber, Time variations of low-rigidity cosmic rays during the recent sunspot cycle, in: J.G. Wilson, S.A. Wouthuysen (Eds.), Progress in Elementary Particle and Cosmic Ray Physics, Vol. 6, North Holland, Amsterdam, 1962, pp. 77-243.

[11] P. Rothwell, J. Quenby, Cosmic rays in the Earth's magnetic field, Nuovo Cimento VIII, Serie X (1958) 249-256.

[12] M.A. Shea, D.F. Smart, L.C. Gentile, Estimating cosmic ray vertical cutoff rigidities as a function of the McIlwain L-parameter for different epochs of the geomagnetic field, Phys. Earth Planet. Int. 48 (1987) 200-205.

[13] J.A. Simpson, W. Fonger, S.B. Treiman, Cosmic radiation intensity-time variations and their origin. I. Neutron intensity variation method and meteorological factors, Phys. Rev. 90 (1953) 934-950.

[14] R.T. Merrill, M.W. McElhinny, P.L. McFadden, The Magnetic Field of the Earth, Academic Press, San Diego, CA, 1998, 531 pp. 
[15] P. Rothwell, Cosmic rays in the Earth's magnetic field, Phil. Mag. 3 (1958) 961-970.

[16] P.J. Kellogg, M. Schwartz, Theoretical study of the cosmic ray equator, Nuovo Cimento XIII (1959) 762-768.

[17] A.E. Sandström, Cosmic ray soft component measurements during a flight from Scandinavia across the North Pole and around Asia and Europe, Nuovo Cimento VIII, Serie X, Suppl. (1958) 263-276.

[18] M. Teucher, Die Absorption der Nukleonenkomponente der kosmischen Strahlung in Luft zwischen Seehöhe und 4000 m, Z. Nat.forsch. 7a (1952) 61-63.

[19] W.W. Brown, Cosmic-ray nuclear interactions in gases, Phys. Rev. 93 (1954) 528-534.

[20] J.A. Lockwood, H.E. Yingst, Correlation of meteorological parameters with cosmic-ray intensities, Phys. Rev. 104 (1956) 1718-1722.

[21] V.C. Tongiorgi, On the mechanism of production of the neutron component of the cosmic radiation, Phys. Rev. 76 (1949) 517-526.

[22] J.G. Roederer, Über die Absorption der Nukleonenkomponente der kosmischen Strahlung in $-21^{\circ}$ geomagnetischer Breite, Z. Nat.forsch. 7a (1952) 765-771.

[23] K.R. Dixit, The statistics of 29000 stars observed in nuclear emulsions in Kenya, Z. Nat.forsch. 10a (1955) 339341.

[24] E. Schopper, E. Lohrmann, G. Mauck, Nukleonen in der Atmosphäre, in: S. Flügge (Ed.), Handbuch der Physik, Vol. XLVI/2, Springer, Berlin, 1967, pp. 373-550.

[25] J.A. Simpson, R.B. Uretz, Cosmic-ray neutron production in elements as a function of latitude and altitude, Phys. Rev. 90 (1953) 44-50.

[26] L. Voyvodic, E. Pickup, Multiple scattering of fast particles in photographic emulsions, Phys. Rev. 85 (1952) 91100.

[27] D.A. Tidman, The mean energy of three-track nuclear disintegrations produced by cosmic-ray particles, Phil. Mag. 43 (1952) 992-993.

[28] M. Teucher, Über die Absorption der Nukleonenkomponente der kosmischen Strahlung in Luft, Helv. Phys. Acta 26 (1953) 434.

[29] J. Masarik, R.C. Reedy, Terrestrial cosmogenic-nuclide production systematics calculated from numerical simulations, Earth Planet. Sci. Lett. 136 (1995) 381-395.

[30] J. Masarik, R.C. Reedy, Monte Carlo simulation of insitu produced cosmogenic nuclides, Radiocarbon 38 (1996) 163-164.

[31] D. Lal, In situ-produced cosmogenic isotopes in terrestrial rocks, Annu. Rev. Earth Planet. Sci. 16 (1988) 355-388.

[32] K. Nishiizumi, E.L. Winterer, C.P. Kohl, J. Klein, R. Middleton, D. Lal, J.R. Arnold, Cosmic ray production rates of ${ }^{10} \mathrm{Be}$ and ${ }^{26} \mathrm{Al}$ in quartz from glacially polished rocks, J. Geophys. Res. 94 (1989) 17907-17915.
[33] E.T. Brown, D.L. Bourlès, F. Colin, G.M. Raisbeck, F. Yiou, S. Desgarceaux, Evidence for muon-induced production of 10Be in near surface rocks from the Congo, Geophys. Res. Lett. 22 (1995) 703-706.

[34] B. Heisinger, M. Niedermayer, F.J. Hartmann, G. Korschinek, E. Nolte, G. Morteani, S. Neumaier, C. Petitjean, P. Kubik, A. Synal, S. Ivy-Ochs, In-situ production of radionuclides at great depth, Nucl. Instr. Methods B 123 (1997) 341-346.

[35] B. Rossi, Interpretation of cosmic ray phenomena, Rev. Mod. Phys. 20 (1948) 537-583.

[36] M.A. Pommerantz, A.E. Sandström, D.C. Rose, Shipboard neutron monitor determination of cosmic ray equator, Nuovo Cimento VIII (Suppl.) (1958) 258-262.

[37] M. Kodoma, Latitude effect of cosmic ray nucleon and meson components at sea level, Nuovo Cimento VIII (Suppl.) (1958) 283-284.

[38] S. Skorka, Breiteneffekt der Nukleonen- und Mesonenkomponente der Ultrastrahlung in Meereshöhe im Indischen und Atlantischen Ozean, Z. Phys. 151 (1958) 630645.

[39] J.A. Lockwood, Variations in the cosmic-ray nucleonic intensity, Phys. Rev. 112 (1958) 1750-1758.

[40] R.A. Langel, D.R. Bararclough, D.J. Kerridge, V.P. Golovkov, T.J. Sabaka, R.H. Estes, Definitive IGRF models for 1945, 1950, 1955, and 1960, J. Geomag. Geoelectr. 40 (1988) 645-702.

[41] T.J. Sabaka, R.A. Langel, R.T. Baldwin, J.A. Conrad, The geomagnetic field 1900-1995, including the large-scale field from magnetospheric sources and the NASA candidate models for the 1995 revision of the IGRF, J. Geomag. Geoelectr. 49 (1997) 157-206.

[42] S.J. McLean, K.D. Meyers, L.D. Morris, W.M. Davis, A Report on Geomagnetic Observatories, 1995, WDC-A NOAA, Boulder, CO, 1995, 110 pp.

[43] D.C. Rose, J. Katzman, The geomagnetic latitude effect on the nucleon and meson component of cosmic rays at sea level, Can. J. Phys. 34 (1956) 1-19.

[44] J.M. Quinn, R.J. Coleman, D.L. Shiel, J.M. Nigro, The joint US/UK 1995 epoch world magnetic model, Naval Oceanographic Office, http://geomag.usgs.gov/Freeware/ geomagix.htm.

[45] G.R. McGregor, S. Nieuwolt, Tropical Climatology, Wiley, Chichester, 1998, $339 \mathrm{pp}$.

[46] J.V. Iribane, W.L. Godson, Atmospheric Thermodynamics, D. Reidel, Dordrecht, 1992, 259 pp.

[47] H. Riehl, Climate and Weather in the Tropics, Academic Press, London, 1979, 611 pp.

[48] Anonymous, International Station Meteorological Climate Summary, Federal Climate Complex, Ashville, 1996. 